\title{
Chemotactic Activity and Receptor Binding of Neutrophil Attractant/Activation Protein-1 (NAP-1) and Structurally Related Host Defense Cytokines: Interaction of NAP-2 With the NAP-1 Receptor
}

\author{
Edward J. Leonard, Teizo Yoshimura, Antal Rot, Kathleen Noer, Alfred Walz, \\ Marco Baggiolini, Daniel A. Walz, Edward J. Goetzl, and C. William Castor \\ Immunopathology Section, Laboratory of Immunobiology, National Cancer Institute, Frederick, Maryland (E.J.L., T.Y.); Sandoz \\ Forschungsinstitut, Vienna, Austria (A.R.); Program Resources, Inc., Frederick, Maryland (K.N.); Theodor-Kocher-Institut, University of \\ Bern, Bern, Switzerland (A.W., M.B); Department of Physiology, Wayne State University School of Medicine, Detroit (D.A.W.); \\ Department of Medicine, University of California School of Medicine, San Francisco (E.J.G.); Department of Internal Medicine, University \\ of Michigan Medical School, Ann Arbor, (C.W.C.)
}

\begin{abstract}
Neutrophil attractant/activation protein-1 (NAP-1) has sequence similarity to platelet factor-4 (PF-4) and to NAP-2 (a truncated form of connective tissue activating protein-III [CTAP-III(des 1-15)]. We compared chemotactic activity for neutrophils of these related proteins. We also included for comparison CTAP-III, CTAP-III(des 1-13), the C-terminal dodecapeptide of PF-4 [PF-4(59-70)], and C5a. Chemotactic potency (EC $\left.{ }_{50}\right)$ was highest for NAP-1 and C5a. Although chemotactic efficacy (peak percentage of neutrophils migrating) was comparable for C5a, NAP-1, and NAP-2, the NAP-2 response occurred only at concentrations 100 -fold higher than the NAP-1 EC Fo $_{50}$ of $10^{-8} \mathrm{M}$. Data for the CTAP-III proteins confirmed that CTAP-III is not an attractant and that chemotactic activity appears as a result of cleavage of residues at the $\mathrm{N}$-terminus to make CTAP-III(des 1-13) or NAP-2 [CTAP-III(des 1-15)]. Chemotactic activity of PF-4 was low and variable, with no significant response by neutrophils from six of nine subjects. In contrast, PF-4(59-70) regularly induced high chemotactic responses, although the $E_{50}$ of $1.6 \times 10^{-5} \mathrm{M}$ was 1,000-fold greater than that of NAP-1. The binding of fluoresceinated NAP-1 to neutrophils was inhibited by unlabeled NAP-1 or NAP-2 but not by PF-4 or PF-4 (59-70). This suggests that NAP-2 interacts with the neutrophil NAP-1 receptor. Despite the low chemotactic potency of NAP-2, it is a potential attractant at sites of injury because of the relatively large amounts of the parent CTAP-III released from platelets, as indicated by a serum concentration of approximately $10^{-6} \mathrm{M}$.
\end{abstract}

Key words: platelet factor-4, PF-4, connective tissue activating peptide-III, CTAP-III, interleukin-8, platelet, flow cytometry

\section{INTRODUCTION}

Sequence similarity has recently led to the assignment of several small protein cytokines to a single family, thought to participate in the response to injury [1-4]. These proteins make up single chains with molecular masses in the 8,000-10,000 dalton range. A distinguishing feature is the occurrence of four half-cystine residues in the chain. When the sequences of the different proteins are appropriately aligned, the proteins fall into two subgroups according to the positions of the first two half-cystines (C-X-C or C-C), and all four half-cystine residues are in register. The $\mathrm{C}-\mathrm{X}-\mathrm{C}$ subgroup includes platelet factor-4 (PF-4), connective tissue activating peptide-III (CTAP-III), and NAP-1 (also known as interleukin-8). PF-4 and CTAP-III are platelet proteins, which are released from $\alpha$-granules when platelets are activated. The biological role of PF-4 is not well estab- lished. Reported activities include heparin binding [5], chemotactic attraction of neutrophils [6], release of mast cell granules [7], stimulation of neutrophil elastase [8], and inhibition of collagenase [9]. CTAP-III is mitogenic for fibroblasts. It also stimulates hyaluronic acid and sulfated glycosaminoglycan synthesis, glucose transport, and prostaglandin $E_{2}$ release [10]. NAP-1 was first identified as a product of lipopolysaccharide (LPS)stimulated monocytes [11]. It is a chemoattractant for neutrophils, and also stimulates the metabolic burst [1]. The latest addition to the C-X-C family is called NAP-2, since, like NAP-1, it is a neutrophil activator and attractant $[12,13]$. It is a truncated form of CTAP-III,

Received April 26, 1990; accepted July 15, 1990.

Reprint requests: Edward J. Leonard, NCI-FCRF, Building 560, Room 12-71, Frederick, MD 21701. 
derived by cleavage of the first $15 \mathrm{~N}$-terminal residues $[12,14,15] .^{1}$

We had two objectives in the experiments described in this communication. The first was to compare chemotactic potency and efficacy of the above $\mathrm{C}-\mathrm{X}-\mathrm{C}$ proteins, to establish a basis for evaluating their significance in vivo. We also included C5a and the C-terminal dodecapeptide of PF-4 [PF-4(59-70)] in this study. C5a is of interest in relation to platelet activation, since C5-derived chemotactic activity can be generated by platelet enzymes released at the site of tissue injury [16]. The chemotactic potency and efficacy of PF-4(59-70) for neutrophils was reported to be comparable to that of the native molecule [17]. This finding may have relevance to other C-X-C proteins, in light of the recent nuclear magnetic resonance study of NAP-1, which suggests that the receptor-binding portion of the molecule is a $\mathrm{C}$ terminal $\alpha$ helix [18]. The second objective, relating to the structural similarity of NAP-1, NAP-2, and PF-4, was to determine if these agonists interacted with the same neutrophil receptor. In addition to determining how much genetic information is devoted to formation of distinct chemoattractant receptors on neutrophils, the question has a new focus because of the possibility noted above that the receptor-interacting portion of NAP-1 and PF-4 is the C-terminal $\alpha$ helix. Agonist binding was evaluated by determining the capacity of unlabeled ligands to inhibit binding of NAP-1-fluorescein isothiocyanate (FITC) to human neutrophils.

\section{MATERIALS AND METHODS Reagents}

All protein solutions used in this study were distributed in aliquots sufficient for single experiments, quick-frozen in liquid nitrogen, and stored at $-80^{\circ} \mathrm{C}$. Recombinant human C5a was a gift from Henry Showell (Pfizer Central Research, Groton, CT). Human NAP-1 was purified to homogeneity from culture fluid of LPSstimulated monocytes [19]. Recombinant NAP-1 was prepared by Dainippon (Osaka, Japan) [20]. Fluoresceinated NAP-1 (NAP-1-FITC) was prepared as previously described [21]. NAP-2 was purified to homogeneity (as judged by silver-staining of SDS-PAGE gels and sequencing of the $\mathrm{N}$-terminus) from platelet-contaminated human monocytes [12]. CTAP-III and CTAP-III(des 1-13) were purified from outdated platelets as recently described [14]. We used two preparations of PF-4 purified to homogeneity from human platelets, one from

'CTAP-III(des 1-15) is CTAP-III without the first $15 \mathrm{~N}$-terminal residues. It is also called neutrophil attractant/activation protein-2 (NAP-2). The former nomenclature is structural, the latter functional. Both terms are useful, depending on the context. In this paper on functional comparison with NAP-1, we use the NAP-2 terminology.
D. Walz [22] and one from A. Walz [13]. The C-terminal dodecapeptide of PF-4, PF-4(59-70), was a synthetic product [7]. Horse type VI cytochrome-c was from Sigma (St. Louis, MO).

\section{Measurement of Chemotactic Responses}

Neutrophils were obtained by dextran sedimentation of heparinized venous blood from normal human subjects. We added $7 \mathrm{ml}$ of 5\% dextran T-500 (Pharmacia-LKB) to $20 \mathrm{ml}$ of human blood. After $40 \mathrm{~min}$, about $12 \mathrm{ml}$ of supernatant plasma was removed and centrifuged. Erythrocytes in the neutrophil pellet were lysed by the addition of $4 \mathrm{ml}$ of $4^{\circ} \mathrm{C} 0.2 \% \mathrm{NaCl}$, followed immediately by $4 \mathrm{ml}$ of $1.6 \% \mathrm{NaCl}$. After centrifugation, the pellet was washed twice with cold Ca-Mg-free Hanks' balanced salt solution. The washed leukocytes, at least $90 \%$ neutrophils, were resuspended in $3 \mathrm{ml}$ of Ca-Mg-free Hanks' solution and stored in ice until use within $60-90 \mathrm{~min}$. As soon as the neutrophils were isolated, serial dilutions of agonists were made in $22^{\circ} \mathrm{C}$ Hanks' solution, with or without $50 \mu \mathrm{g} / \mathrm{ml}$ cytochrome-c. Bottom wells of multiwell chemotaxis chambers [23] were filled in duplicate with serial dilutions of attractants, and the chambers were assembled, with 3- $\mu \mathrm{m}$-pore-diameter polyvinylpyrrolidone (PVP)-free polycarbonate membranes separating upper cell wells from lower attractant wells. The cold stock suspension of neutrophils was diluted at least tenfold with $22^{\circ} \mathrm{C}$ Hanks' solution, with or without 50 $\mu \mathrm{g} / \mathrm{ml}$ cytochrome-c, to a final concentration of $7 \times 10^{5}$ neutrophils $/ \mathrm{ml}$. After addition of $50 \mu \mathrm{l} /$ well, chambers were incubated at $37^{\circ} \mathrm{C}$ in humidified air with $5 \% \mathrm{CO}_{2}$ for $45 \mathrm{~min}$. The polycarbonate filter was then removed from the chamber, nonmigrated cells were wiped off the upper surface, and the filter was air-dried and stained with Diff-Quik (American Scientific Products, McGaw Park, IL).

Migrated neutrophils were examined microscopically with a $\times 25$ objective and an image analyzer. Three randomly chosen fields per well were surveyed (a total of $0.48 \mathrm{~mm}^{2}$ of the $8 \mathrm{~mm}^{2}$ well area). The image analyzer can count individual cells or determine the total area occupied by stained cells. Since the former underestimates cell number if migrated cells touch one another, we determine migrated neutrophil area and converted to number of neutrophils by dividing by an average area per neutrophil.

\section{Inhibition of NAP-1-FITC Binding by Unlabeled Ligand}

This was done exactly as described elsewhere [21]. Fixed concentrations of NAP-1-FITC were mixed with increasing concentrations of unlabeled ligand and equilibrated with neutrophils at $0^{\circ} \mathrm{C}$. The cells were then washed and surveyed for bound fluorescence by flow cytometry. 


\section{RESULTS}

\section{Chemotactic Responses to the Different Ligands}

A representative response of neutrophils to serial fivefold dilutions of three different chemoattractants is shown in Figure 1A. The chemotactic response as a function of NAP-1 or C5a rose to a peak and then declined. This is typical of pure chemoattractants, provided that there is sufficient solubility and amount of attractant to cover the appropriate concentration range. The number of neutrophils responding to NAP-2 was comparable to the NAP-1 or C5a response, but higher concentrations of NAP-2 were required. Figure 1B shows that PF-4(59-70) also caused neutrophil migration, but only at concentrations that were about three orders of magnitude higher than the NAP-1 optimum.

Pooled data for this part of the study are shown in Table 1 . The $\mathrm{EC}_{50}$ potency ratio column shows that $\mathrm{C} 5 \mathrm{a}$ and NAP-1 are much more potent neutrophil attractants than the other proteins listed. Although efficacy (peak percentage migration) of NAP-2 was similar to that of NAP-1, the $\mathrm{EC}_{50}$ was two orders of magnitude greater. The limited supply of CTAP-III(des 1-13) restricted us to two chemotaxis experiments, which suggest potency and efficacy comparable to that of NAP-2 (CTAP-III(des 1-15)]. The chemotactic response to CTAP-III (two experiments) was undetectable or minimal, confirming published results [13].

Among all agonists tested, PF-4(59-70) caused the highest neutrophil migration response, but the $\mathrm{EC}_{50}$ potency ratio was the lowest. Despite the high concentrations required, the response to PF-4(59-70) was primarily chemotactic, not chemokinetic (Table 2). The first column of Table 2 shows that PF-4(59-70) does not induce chemokinesis, since there is only a minimal increase in migration as a function of increasing concentrations of agonist in the cell suspension. The migration response to PF-4(59-70) in attractant wells only (Table 2, first row) was inhibited when the positive gradient was eliminated (Table 2, bottom row), which indicates that the response is chemotactic. In contrast to the data for PF-4(59-70), the responses to native PF-4 were low and variable, with no significant effect on neutrophils from six of nine subjects (Table 1). Lack of response to PF-4 occurred at concentrations as high as $1.3 \times 10^{-4} \mathrm{M}$, which is tenfold higher than the $\mathrm{EC}_{50}$ for PF-4(59-70).

\section{Inhibition of NAP-1-FITC Binding by Unlabeled Agonists}

Figure 2A illustrates the capacity of different members of the C-X-C family to compete with NAP-1-FITC for human neutrophil NAP-1 binding sites. NAP-1 was the best competitor, followed by the truncated forms of CTAP-III, and then by native CTAP-III. Figure 2B shows another experiment, with NAP-1 and NAP-2 at comparable concentrations. Neither the C-terminal dodecapeptide of PF-4 (Fig. 2B) nor native PF-4 (data not shown) inhibited NAP-1-FITC binding. The results indicate that NAP-2 interacts with the NAP-1 receptor, whereas PF-4(59-70) does not.

\section{DISCUSSION}

A major objective of this research was to compare chemotactic activity of several members of the C-X-C family of proteins under identical assay conditions. ${ }^{2}$ Assays were done in multiwell chemotaxis chambers with 3- $\mu \mathrm{m}$-diameter pore size, $10-\mu \mathrm{m}$-thick PVP-free polycarbonate membranes. This method lends itself to quantitative evaluation, since many assay wells can be set up in a short time, and migration data can be generated by image analysis. The polycarbonate membrane should be distinguished from the $150-\mu \mathrm{m}$-thick cellulosic filters used by some investigators in classical studies on neutrophil chemotaxis [26]. We were concerned about the possibility of loss of low concentrations of protein agonists on the walls of dilution tube or chemotaxis well. The total amount of protein lost from a solution is limited by the adsorptive capacity of the container. Since the percentage loss is negligible at protein concentrations of $50 \mu \mathrm{g} / \mathrm{ml}$ or higher [27], loss of agonist can be prevented by dilution in a $50 \mu \mathrm{g} / \mathrm{ml}$ solution of a nonreactive protein. We found that addition of cytochrome-c to the Hanks' solution used for diluting attractants and preparing neutrophil suspensions did not affect neutrophil random migration and sometimes improved attractant

\footnotetext{
${ }^{2}$ Methods for measurement of neutrophil chemotaxis differ greatly, which probably accounts for disparate reports on activity of the same agonist. Each method may have advantages, but, for comparison of different agonists, one method should be selected. As noted, we use a $10-\mu \mathrm{m}$-thick polycarbonate membrane with $3 \mu \mathrm{m}$ holes, through which neutrophils can migrate toward the attractant. The number of migrated neutrophils can be quantified, since they adhere to the attractant side of the membrane. However, if we use a membrane coated with PVP, or if bovine serum albumin (BSA) is added to the medium, 20-50\% of the migrated neutrophils fall off into the attractant well [24]. Since migrated neutrophils are variably distributed between membrane and attractant well fluid in the presence of BSA, counts of either membrane-adherent or well fluid neutrophils will underestimate the total migrated number, and this will affect calculated efficacy as well as the shape of the dose-response curve. When a $150-\mu \mathrm{m}$-thick cellulosic filter is used for chemotaxis assays, the response is frequently quantified by the leading front method, which measures the distance traveled by the two neutrophils that migrated the farthest into the filter. By this method, for example, NAP-1 activity was detected at $10^{-10} \mathrm{M}$ [25]. However, this figure cannot be related to our data in Table 1, since leading front data provide no information about the concentration of agonist required for migration of the bulk of the neutrophils or about chemotactic efficacy (percentage of input neutrophils migrating). In the study of Harvath et al. [24], detectable neutrophil migration occurred at $10^{-10} \mathrm{M}$ fMLP, whereas peak efficacy requires $10^{-6} \mathrm{M}$.
} 


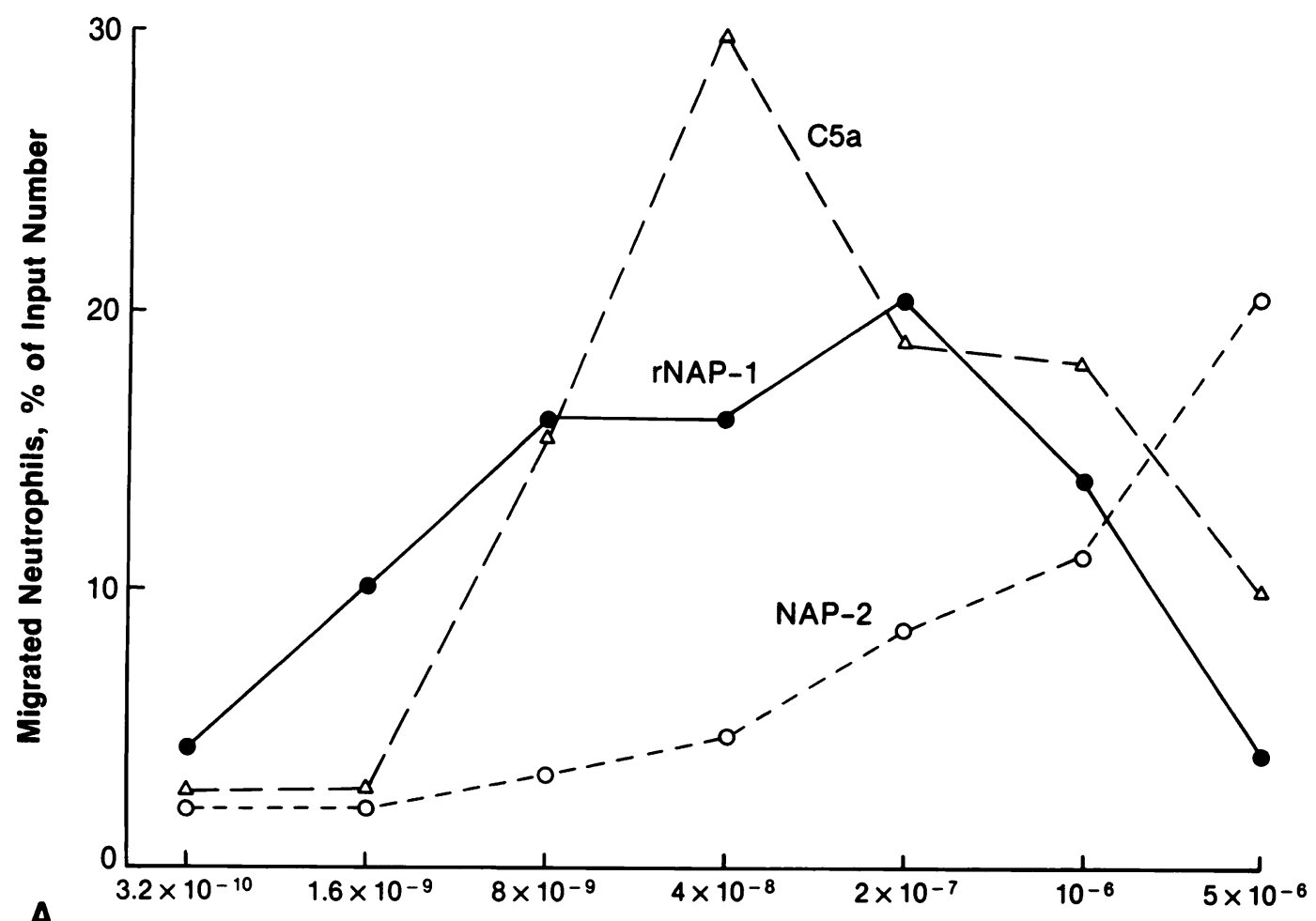

Molar Concentration (logarithmic scale)

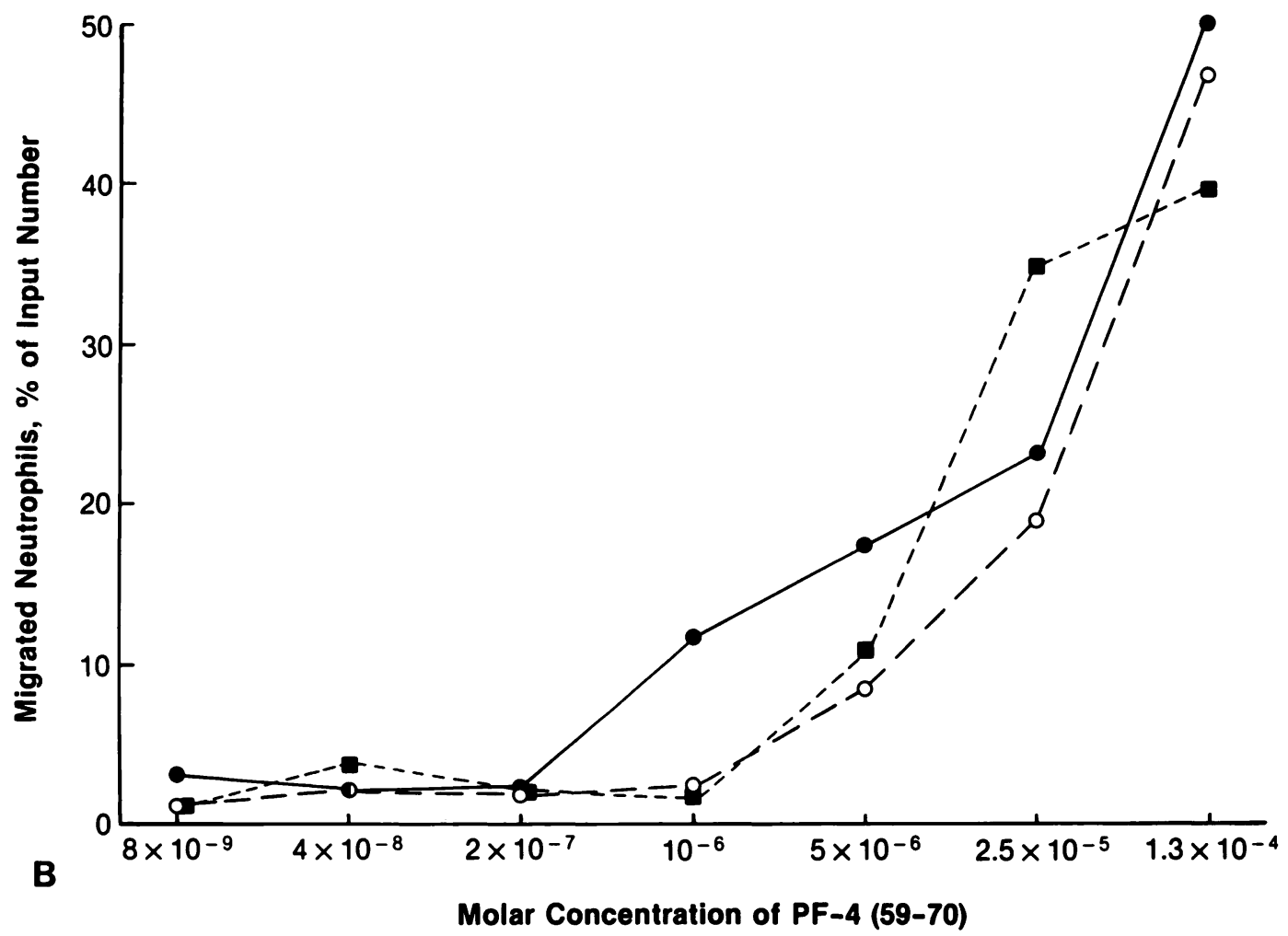

Fig. 1. A: Migration response of human neutrophils to NAP-1, NAP-2, and C5a. B: Response of neutrophils from three human subjects to PF-4(59-70). 
TABLE 1. Chemotactic Efficacy and Potency of Attractants

\begin{tabular}{|c|c|c|c|c|}
\hline \multirow{8}{*}{$\begin{array}{l}\text { C5a } \\
\text { NAP-1 } \\
\text { rNAP-1 } \\
\text { NAP-2 } \\
\text { CTAP-III(des } 1-13)^{c} \\
\text { PF-4 } \\
\text { PF-4(59-70) }\end{array}$} & \multirow{8}{*}{$\begin{array}{c}\begin{array}{c}\text { Peak \% } \\
\text { migration }\end{array} \\
33 \pm 5^{b}(6) \\
23 \pm 4(7) \\
23 \pm 1(5) \\
26 \pm 3(7) \\
19 \pm 3(2) \\
19 \pm 3(3)^{d} \\
40 \pm 3(6)\end{array}$} & Molarity at peak & $\mathrm{EC}_{50}$ & \multirow{2}{*}{$\frac{\text { Potency ratio }^{\mathrm{a}}}{1,800}$} \\
\hline & & $1 \pm 0.3 \times 10^{-7}$ & $9 \pm 2 \times 10^{-9}$ & \\
\hline & & $6 \pm 2 \times 10^{-8}$ & $1 \pm 0.4 \times 10^{-8}$ & 1,600 \\
\hline & & $\times 10^{-7}$ & $4.2 \pm 1 \times 10^{-9}$ & 3,800 \\
\hline & & $\times 10^{-6}$ & $1.2 \pm 0.2 \times 10^{-6}$ & 13 \\
\hline & & $\times 10^{-6}$ & $1.4 \pm 0.6 \times 10^{-6}$ & 11 \\
\hline & & $\times 10^{-6}$ & $1.3 \pm 0.3 \times 10^{-6}$ & 12 \\
\hline & & $\times 10^{-4}$ & $1.6 \pm 0.5 \times 10^{-5}$ & 1 \\
\hline
\end{tabular}

${ }^{\text {aReciprocal of }} \mathrm{EC}_{5}$ agonist/EC 50 PF4(59-70).

'SEM for indicated number of experiments (in parentheses) with different subjects.

'CTAP-III (not truncated) was tested twice. There was no response with one subject; the $8 \%$ migration peak at $5 \times 10^{-6} \mathrm{M}$ with the other was not necessarily significant, since there was polyethylene glycol in the CTAP-III, which causes neutrophil migration.

${ }^{d}$ Among six other donors tested with PF-4, there was no response in four, neutrophil clumping in one, and very low response without a dose-response trend in one. One of two lots of PF-4 (purified by A. Walz or D. Walz) was used in these experiments. Each lot was represented among both responders and nonresponders.

TABLE 2. Response to Combinations of PF-4(59-70) in Cell and Attractant Wells

\begin{tabular}{lccccc}
{$\left[\begin{array}{c}5 \\
\text { in cell well }\end{array}\right.$} & \multicolumn{5}{c}{$[\mathrm{PF}-4(59-70)](\mathrm{M})$ in attractant well } \\
\cline { 2 - 6 } & 0 & $10^{-6}$ & $5 \times 10^{-6}$ & $2.5 \pm 10^{-5}$ & $1.3 \times 10^{-4}$ \\
\hline 0 & $0.7^{\mathrm{a}}$ & 0.5 & 4.2 & 10.8 & 10.4 \\
$10^{-6}$ & 0.6 & 1.4 & 5.9 & 6.4 & 8.1 \\
$5 \times 10^{-6}$ & 0.7 & 0.6 & 1.9 & 6.0 & 7.1 \\
$2.5 \times 10^{-5}$ & 1.1 & 2.2 & 3.7 & 7.0 & - \\
$1.3 \times 10^{-4}$ & 2.0 & 1.4 & 1.4 & 1.2 & 3.4 \\
0 & 0.9 & 2.5 & 5.0 & 7.5 & 11.7 \\
$10^{-6}$ & 1.0 & 2.1 & 6.2 & 10.6 & 8.6 \\
$5 \times 10^{-6}$ & 1.3 & 1.1 & 4.6 & 7.5 & 8.9 \\
$2.5 \times 10^{-5}$ & 1.6 & 2.1 & 3.4 & 11.5 & 5.9 \\
$1.3 \times 10^{-4}$ & 0.8 & 1.4 & 1.5 & 3.7 & 6.4 \\
\hline
\end{tabular}

aMigrated neutrophils per well $\times 10^{-3}$. Input neutrophil number was 35,000 . All cell suspensions and attractants were in $50 \mu \mathrm{g} / \mathrm{ml}$ cytochrome-c in Hanks' balanced salt solution. Data are from experiments with neutrophils from two different human subjects.

potency by one serial dilution step at low concentrations of agonist. We avoided BSA, because of the variable effects of this protein on random migration and chemotactic responses.

Our results show that chemotactic potency and efficacy of NAP-1 and C5a for neutrophils are comparable. We confirm a report by A. Walz et al. [13] that NAP-2 is a neutrophil attractant, with efficacy comparable to that of NAP-1. In contrast to that report, we find that NAP-2 potency is lower than that of NAP-1. Consistent with our results is the fact that NAP-2 is not as effective as NAP-1 in competing with NAP-1-FITC for binding sites on neutrophils (Fig. 2, Table 3). The chemotaxis assay used by Walz et al. [13] differs from ours (BSA in the medium, longer incubation, collection of migrated cells that do not adhere to the membrane, and much higher random migration). It is likely that the observed differ- ences in NAP-2 potency relate to a methodological detail of the chemotaxis assay, since the NAP-2 in both studies was purified by $\mathrm{A}$. Walz.

The C-terminal dodecapeptide of PF-4 also caused neutrophil migration, although responses comparable in magnitude to that of NAP-1 or C5a required 1,000-fold higher concentrations. Whereas NAP-1 and NAP-2, which have considerable sequence similarity, appear to interact with the NAP-1 neutrophil receptor, PF-4(59-70) does not. The chemotactic activity of this C-terminal portion of PF-4 is of great interest in light of the recent nuclear magnetic resonance study [18] of NAP-1 in solution, suggesting that a $C$-terminal $\alpha$ helix, mounted on a $\beta$ sheet formed by residues $56-72$, is the part of the molecule that reacts with the receptor. However, PF-4 preparations from two different sources had minimal chemotactic activity in our study. This confirms results 

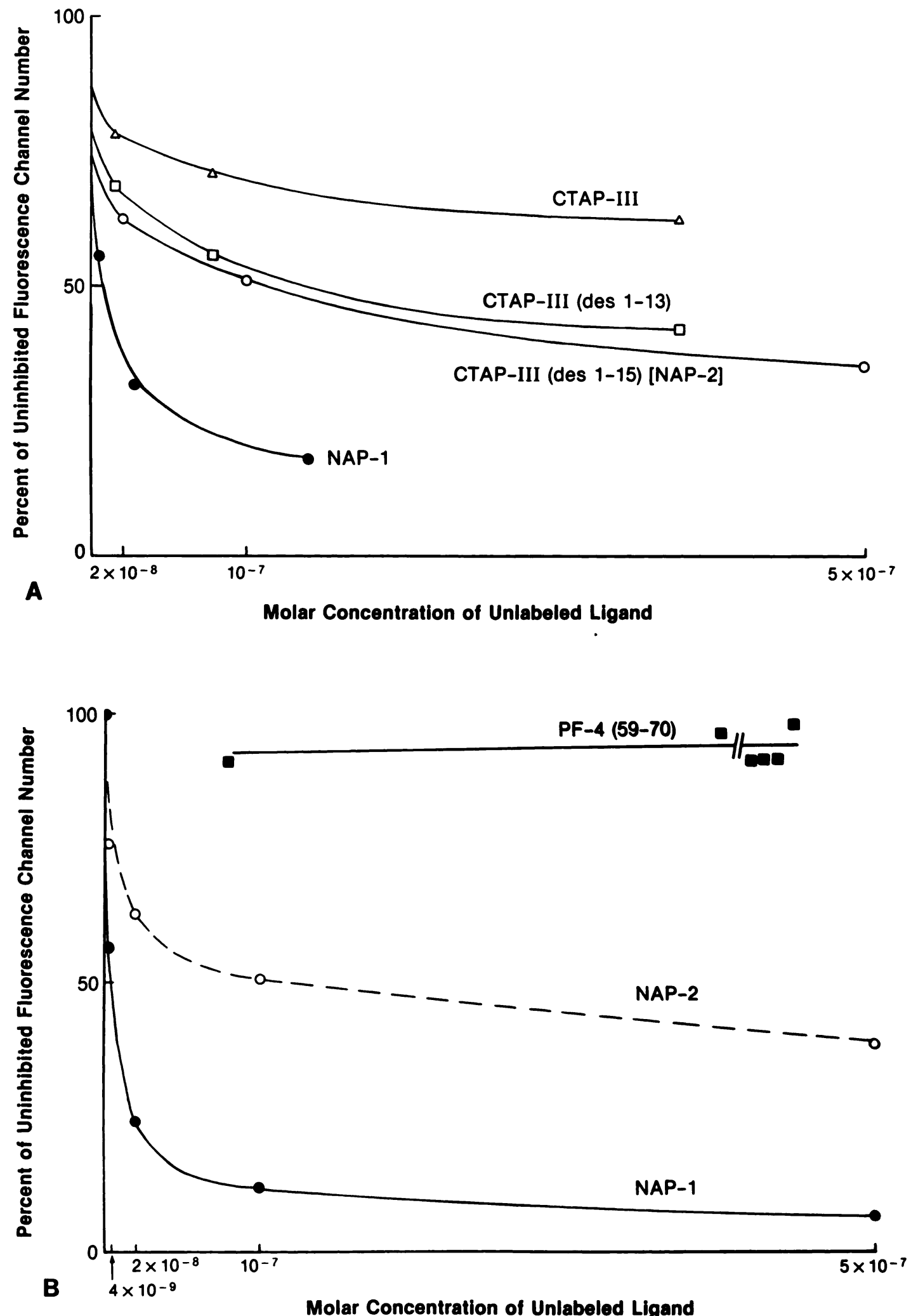

Fig. 2. Inhibition of binding of NAP-1-FITC to human neutrophils by unlabeled ligands. A: CTAP-III, CTAP-III(des 1-13), CTAP-III(des 1-15) [NAP-2], and NAP-1. B: PF-4(59-70), NAP-2, NAP-1. The 4 PF-4 symbols after the break represent fivefold increasing concentrations up to $1.3 \times 10^{-3} \mathrm{M}$. The inhibition curves are the result of mixing unlabeled ligand with $2 \times 10^{-7} \mathrm{M}$ NAP-1-FITC. Data are mean fluorescence channel number minus autofluorescence channel number, expresesd as a percentage of the value obtained with $2 \times 10^{-7}$ M NAP-1-FITC. 
TABLE 3. Inhibition of NAP-1-FITC Binding by Unlabeled Ligand

\begin{tabular}{lccc}
\hline Ligand & $\begin{array}{c}\text { Percent } \\
\text { inhibition }^{\mathrm{a}}\end{array}$ & IC $_{50}$ & Potency ratio \\
\hline NAP-1 (4) & $90 \pm 3$ & $2 \pm .6 \times 10^{-8}$ & 10 \\
NAP-2 (4) & $69 \pm 5$ & $8 \pm 3 \times 10^{-8}$ & 3 \\
CTAP-III(des 1-13)(1) & 58 & $2 \times 10^{-7}$ & 1 \\
CTAP-III (2) & $33 \pm 5$ & $-^{c}$ & - \\
\hline
\end{tabular}

a Maximal inhibition observed with different cuncentrations of unlabeled ligand mixed with $2 \times 10^{-7}$ M NAP-1-FITC. IC 50 is molarity of unlabeled ligand required for $50 \%$ inhibition. Potency ratio for ligand is IC 50 of CTAP-IIldes $(1-13) /$ IC $_{50}$ of ligand.

${ }^{b}$ Numbers of experiments are in parentheses.

'Did not reach $50 \%$ inhibition.

obtained by Walz et al. [13] with one of the preparations, but does not agree with previous reports on the chemotactic activity of PF-4 for neutrophils in the $10^{-7}-10^{-5} \mathrm{M}$ range $[6,17]$. Since the chemotactic activity of PF$4(59-70)$ suggests that neutrophils may have a receptor for this ligand, additional experiments with purified native PF-4 are warranted.

In light of our comparative study, it is of interest to speculate about the generation and role of chemoattractants in tissue injury. Stored proteins are released from platelet $\alpha$ granules in the initial response and this can lead to formation of two chemoattractants from protein precursors. Secreted platelet products can activate $\mathrm{C} 5$ to become a chemoattractant [16]. The mechanism is probably similar to the cleavage by trypsin of a C-terminal peptide of the $\alpha$ chain of $\mathrm{C} 5$, which induces chemotactic activity even though the split products remain attached to one another via a disulfide bond [28]. Platelet CTAP-III becomes the NAP-2 chemoattractant when a 15-residue fragment is cleaved from the $\mathrm{N}$-terminus. Porcine elastase [14] and enzymes in human monocyte culture fluid [15] are capable of this cleavage If neutrophil elastase can also cleave CTAP-III, there is a potential for positive feedback generation of NAP-2. It should also be noted that serum concentrations of CTAP-III are in the range $5-35 \mu \mathrm{g} / \mathrm{ml}$, which suggests that there would be considerable CTAP-III substrate available at local sites of platelet aggregation. Thus the lower potency of NAP-2 relative to C5a or NAP-1 may not be a biological limitation. In summary, chemotactic attraction of neutrophils to sites of tissue injury could be mediated early by enzymatic activation of two precursors, C5 and CTAP-III. The products-activated C5 and NAP-2engage C5a and NAP-1 receptors. Since NAP-1 is not preformed, it would appear later, after a stimulus such as LPS or interleukin-1 causes synthesis and secretion by cells at the site of injury. The time from production stimulus to secretion is short; NAP-1 was detected in human lung macrophage culture fluids as early as $6 \mathrm{hr}$ after stimulation by LPS [29].

\section{ACKNOWLEDGMENTS}

E.J.G. was supported in part by a research contract from Stimmune Biotechnologies Division of Rainin Instrument Co., Inc. D.A.W. was supported in part by National Institutes of Health grant HL 27073.

\section{REFERENCES}

1. Baggiolini, M., Walz, A., and Kunkel, S.L. Neutrophil-activating peptide-1/interleukin 8 , a novel cytokine that activates neutrophils. J. Clin. Invest. 84,1045, 1989.

2. Leonard, E.J., and Yoshimura, T. Human monocyte chemoattractant protein-1 (MCP-1). Immunol. Today 11,97, 1990.

3. Matsushima, K., and Oppenheim, J.J. Interleukin 8 and MCAF: Novel inflammatory cytokines inducible by IL-1 and TNF. Cytokine 1,2, 1989.

4. Leonard, E.J., and Yoshimura, T. Neutrophil attractant/activation protein-1 [NAP-1 (IL-8)]. Am. J. Respir. Cell Mol. Biol. 2,479, 1990.

5. Handin, R.I., and Cohen, H.J. Purification and binding properties of human platelet factor four. J. Biol. Chem. 251,4273, 1976.

6. Deuel, T.F., Senior, R.M., Chang, D., Griffin, G.L., Heinrikson, R.L., and Kaiser, E.T. Platelet factor 4 is chemotactic for neutrophils and monocytes. Proc. Natl. Acad. Sci. USA 78,4584, 1981.

7. Brindley, L.L., Sweet, J.M., and Goetzl, E.J. Stimulation of histamine release from human basophils by human platelet factor 4. J. Clin. Invest. 72,1218, 1983.

8. Lonky, S.A., and Wohl, H. Stimulation of human leukocyte elastase by platelet factor 4 . Physiologic, morphologic and biochemical effects on hamster lungs in vitro. J. Clin. Invest. $67,817,1981$.

9. Hiti-Harper, J., Wohl, H., and Harper, E. Platelet factor 4: an inhibitor of collagenase. Science 199,991, 1978.

10. Castor, C.W. Regulation of connective tissue metabolism. In Arthritis and Allied Conditions, 11th ed. (McCarty, D.J., Ed.). Philadelphia: Lea \& Febiger, p 242, 1988.

11. Yoshimura, T., Matsushima, K., Oppenheim, J.J., and Leonard, E.J. Neutrophil chemotactic factor produced by lipopolysaccharide (LPS) stimulated human blood mononuclear leukocytes. I. Partial characterization and separation from interleukin-1 (IL-1). J. Immunol. 139,788, 1987.

12. Walz, A., and Baggiolini, M. A novel cleavage product of $\beta$-thromboglobulin formed in cultures of stimulated mononuclear cells activates human neutrophils. Biochem. Biophys. Res. Commun. 159,969, 1989. 
13. Walz, A., Dewald, B., von Tscharner, V., and Baggiolini, M. Effects of the neutrophil-activating peptide NAP-2, platelet basic protein, connective tissue-activating peptide III, and platelet factor 4 on human neutrophils. J. Exp. Med. 170,1745, 1989.

14. Castor, C.W., Walz, D.A., Ragsdale, C.G., Hossler, P.A., Smith, E.M., Bignall, M.C., Aaron, B.P., and Mountjoy, K. Connective tissue activation XXXIII. Biologically active cleavage products of CTAP-III from human platelets. Biochem. Biophys. Res. Commun. 163,1071, 1989.

15. Walz, A., and Baggiolini, M. Generation of the neturophilactivating peptide NAP-2 from platelet basic protein or connective tissue activating peptide III through monocyte proteases. J. Exp. Med. 171,449, 1990.

16. Weksler, B.B., and Coupal, C.E. Platelet-dependent generation of chemotactic activity in serum. J. Exp. Med. 137,1419, 1973.

17. Goldman, D.W., Hannah, A.L., and Goetzl, E.J. Inhibition of human neutrophil receptor-mediated uptake of $\mathrm{N}$-formyl-metleu-phe by platelet factor 4(59-70). Immunology 54,163, 1985.

18. Clore, G.M., Appella, E., Yamada, M., Matsushima, K., and Gronenborn, A.M. The three-dimensional structure of interleukin-8 in solution. Biochemistry 29,1689, 1990.

19. Yoshimura, T., Robinson, E.A., Appella, E., Matsushima, K., Showalter, S.D., Skeel, A., and Leonard, E.J. Three forms of monocyte-derived neutrophil chemotactic factor (MDNCF) distinguished by different lengths of the amino-terminal sequence. Mol. Immunol. 26,87, 1989.

20. Furuta, R., Yamagishi, J., Hiotada, K., Sakamoto, F., Fukui, T., Matsui, Y., Sohmura, Y., Yamada, M., Yoshimura, T., Larsen, C.G., Oppenheim, J.J., and Matsushima, K. Production and characterization of recombinant human neutrophil chemotactic factor. J. Biochem. 106,436, 1989.

21. Leonard, E.J., Skeel, A., Yoshimura, T., Noer, K., Kutvirt, S., and van Epps, D. Leukocyte specificity and binding of human neutrophil attractant/activation protein-1 (NAP-1). J. Immunol. $144,1323,1990$.

22. Ciaglowski, R.E., Snow, J., and Walz, D.A. Isolation and amino acid sequence of bovine platelet factor 4 . Arch. Biochem. Biophys. 250,249, 1986.

23. Falk, W., Goodwin, R.H., Jr., and Leonard, E.J. A 48-well microchemotaxis assembly for rapid and accurate measurement of leukocyte migration. J. Immunol. Methods 33,239, 1980.

24. Harvath, L., Falk, W., and Leonard, E.J. Rapid quantitation of neutrophil chemotaxis: Use of a polyvinylpyrrolidone-free polycarbonate membrane in a multiwell assembly. J. Immunol. Methods 37,39, 1980.

25. Lindley, I., Aschauer, H., Seifert, J.-M., Lam, C., Brunowsky, W., Kownatzki, E., Thelen, M., Peveri, P., Dewald, B., von Tscharner, V., Walz, A., and Baggiolini, M. Synthesis and expression in Escherichia coli of the gene encoding monocytederived neutrophil-activating factor: biological equivalence between natural and recombinant neutrophil-activating factor. Proc. Natl. Acad. Sci. USA 85,9199, 1988.

26. Wilkinson, P.C. Chemotaxis and Inflammation. Edinburgh: Churchill Livingstone, p 33, 1974.

27. Askenase, P.W., and Leonard, E.J. Solid phase radioimmunoassay of beta-1-C globulin. Immunochemistry 7,29, 1970.

28. Wetsel, R.A., and Kolb, W.P. Complement-independent activation of the fifth component (C5) of human complement: Limited trypsin digestion resulting in the expression of biologic activity. J. Immunol. 128,2209, 1982.

29. Rankin, J.A., Sylvester, I., Smith, S., Nolfo, R., and Leonard, E.J. Alveolar macrophages culture in vitro release $\mathrm{LTB}_{4}$ and neutrophil attractant/activation protein-1 [NAP]1 (IL-8)] sequentially in response to stimulation with LPS and zymosan. J. Clin. Invest. 86,1556, 1990. 